\title{
Physical Sensors for Biomedical Applications
}

\author{
(Invited review)
}

\author{
Rafiq Ahmad and Khaled N. Salama \\ Sensors Lab, Electrical Engineering Program, Computer, Electrical and Mathematical Science and Engineering Division, King \\ Abdullah University of Science and Technology (KAUST), Thuwal 23955-6900, Kingdom of Saudi Arabia \\ rafiq.ahmad@kaust.edu.sa(R.Ahmad),khaled.salama@kaust.edu.sa (K.N. Salama)
}

\begin{abstract}
Use of sensors is increasing day-by-day in the real world to improve the quality of life by providing information on medical diagnostics for healthcare. Among numerous emerging sensing technologies, physical sensors "electronic devices" have been successfully demonstrated in the field of biomedical applications because of their excellent operation capability. Physical sensing is a unique sensing platform, where sensing devices are responsive towards physical properties (e.g., radiation, light, flow, heat, pressure, magnetic field, and parameters related to mass or energy) and convert them into signals for quantification. This review paper describes the different physical sensors and their biomedical applications, current main challenges, and future developments.
\end{abstract}

Keywords-physical sensors; electronic devices; healthcare; biomedical applications.

\section{I.}

\section{INTRODUCTION}

In the physical sensing platforms, the physical variables (such as thermal, mechanical, electrical, magnetic, and atomic/ nuclear) associated with the biomedical systems are detected and monitored $[1,2]$. The natural physical properties such as shear, torsion, pressure, temperature, and humidity are the fundamentals for physical sensors that are converted into the signals for measuring the physical quantities using an observer or instrument. The use of physical sensors is not limited to the real-time healthcare monitoring but are also utilized in other advanced applications, such as soft robotics, wearable consumer electronics, electronic skins, and smart medical prosthetics [3]. However, mainly in the biomedical field, there is an increasing demand for developing the effective and implementable sensors.

There has been an increasing attempt to design physical sensing devices with long-term stability, excellent optical transparency, and increased sensitivity, mainly with optimized for particular sensing platform [4-6]. The utilization of materials such as superconductors or nanophase materials, optical fibers and semiconductors micro fabrication technology have guaranteed the high precision, possibility of multifunction, and integration of physical sensors. Sensors developed with different flexible materials and substrate have been designed and implemented for biomedical applications that provide an excellent surface for various topologies and geometries. Notably, flexible physical sensors are attractive because of superior deformability, optical transparency, stretchability, and compliancy.
Physical sensors operate based on relative output signal variations in their electrical parameters. A classification of physical sensors is presented in Fig. 1. These sensors detect and quantify the different physical nature or phenomenon or physical system. Physical sensing devices have been most widely used and made a remarkable contribution to our daily life including agriculture, aerospace, military, and industry development. Recently, nanomaterials such as semiconductors, metals, and polymers have been extensively utilized as active sensing elements to enhance the sensing performance of physical sensors. Such nanomaterials (e.g., carbon nanotubes, metal and metal oxide nanowires, polymers nanofibers, and metal nanoparticles) based physical sensors have been developed for healthcare and medical applications.

This paper provides a review of current research developments on the physical sensors that are mainly utilized in the biomedical applications. This review briefly introduces the physical sensors and discusses the potential biomedical applications followed by conclusions.

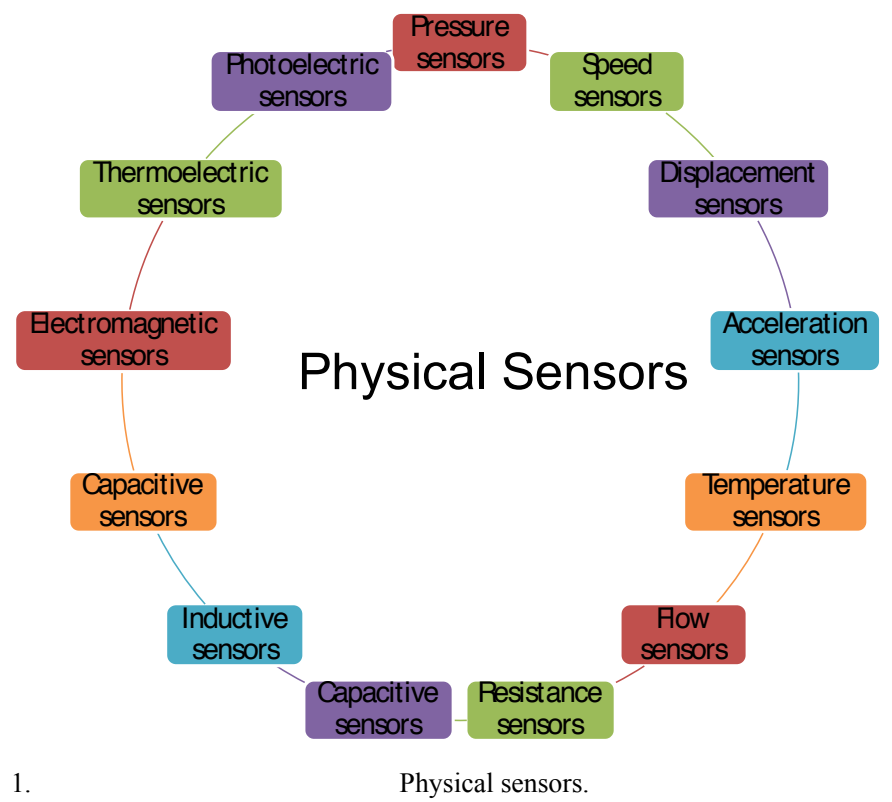




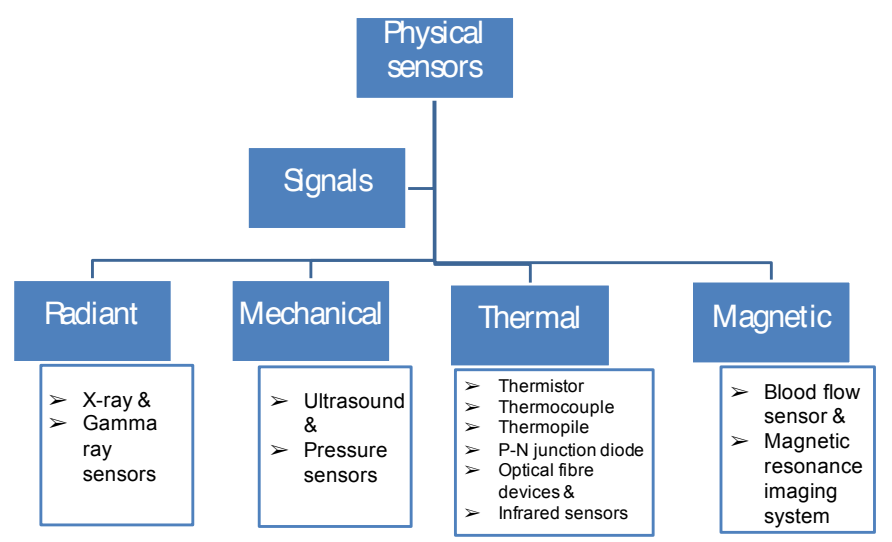

2. Types of physical sensors in biomedical applications based on their signals.

\section{TYPES OF PHYSICAL SENSORS AND BIOMEDICAL APPLICATIONS}

The biomedical physical sensors are divided into four classes based on their signals (Fig. 2). In the class 1, radiation sensors address the X-ray and gamma ray-based sensors. In the class 2, mechanical sensors include ultrasound and pressure sensors. In the class 3 , thermal sensors include a range of sensors such as thermocouple, thermistor, thermopile, optical fibre devices, P-N junction diode, and infrared sensors. Among these thermal sensors, the optical fibre devices are the most promising and offer enormous expectations [7]. In the class 4, magnetic sensors include the important blood flow monitoring sensors and magnetic resonance imaging systems. Brief descriptions of these classes are presented bellow.

\section{A. Radiation Sensors}

Radiation sensors utilize radiations (X-rays or gamma rays) for imaging and treatment in a variety of biomedical applications. In particular, radiation sensors are mostly employed for medical imaging while using ionising radiation. However, there are effects of ionising radiation on health. To overcome danger of ionising radiation, a clear understanding of the benefits and risks associated with radiation and radioactivity are needed. Currently, medical imaging are categorized into three categories based on the ionising radiation.

(i) Medical imaging using X-rays as an external beam source

(ii) Medical imaging using a radiation source (radio-isotope) internal to the body

(iii) Medical imaging without using ionising radiation

For better and precise imaging, combinations of two categories are preferred. For example, combination of images from positron emission tomography (PET) and single photon emission computed tomography (SPECT) measured using radio-pharmaceutical tracers (e.g., computed tomography (CT) or magnetic resonance imaging (MRI)) for position accuracy; combination of $\mathrm{CT}$ and PET but it requires radiation dose before measurements; and combination of PET and MRI has been recently developed. There are still attempts to design sensor systems to lower the cost.

\section{B. Mechanical Sensors}

Mechanical sensors include ultrasound and pressure sensors for biomedical applications. These sensors target a large number of physical variations (e.g., force, mass, strain, pressure, velocity, weight, and acceleration). A detailed physical variables are listed in Table 1 . The ultrasound waves emitted by transducer pass into the tissues and are detected using sensors to monitor health conditions without involving any invasive procedures $[8,9]$. The mechanical sensors rely on the mechanical vibrations, piezoresistive effect, capacitance, triboelectricity, and resistance. Additionally, these sensing components are driven by mechanical deformations (pressing, bending, stretching, and twisting) of devices.

On the other hand, pressure sensors utilize force applied on the piezoelectric material that produce the electric potential. These sensors are required in many fields, such as industrial process controls, biomedical systems, and environmental monitoring. To detect external pressure in human beings, a new capacitive pressure sensor is utilized as non-invasive blood pressure measurements, which had extremely high sensitivity of $2.24 \mu \mathrm{F} / \mathrm{kPa}$ [10]. Also, pressure sensors are used to monitor internal pressure [11].

TABLE 1. PHYSICAL VARIABLES DETECTING SENSORS.

\begin{tabular}{|c|c|c|}
\hline Physical Quantity & Sensor & Variable detected \\
\hline \multirow{3}{*}{ Geometric } & $\begin{array}{c}\text { Linear variable differential } \\
\text { transformer }\end{array}$ & Displacement \\
\cline { 2 - 3 } & Ultrasonic transit time & Displacement \\
\cline { 2 - 3 } & Strain gauge & Strain \\
\hline \multirow{2}{*}{ Force-torque } & Load cell & $\begin{array}{c}\text { Applied force/ } \\
\text { torque }\end{array}$ \\
\hline \multirow{2}{*}{ Kinematic } & Accelerometer & $\begin{array}{c}\text { Acceleration } \\
\text { Velocimeter }\end{array}$ \\
\cline { 2 - 3 } Thermal & Thermometer & Telocity \\
\hline \multirow{2}{*}{ Fluidic } & Thermal flux sensor & Heat flux \\
\cline { 2 - 3 } & Flow meter & Flow \\
\hline \multirow{2}{*}{ Pressure transducer } & Pressure \\
\hline
\end{tabular}

\section{Thermal Sensors}

Thermal sensors are utilized to measure the on-body temperature measurements, which is a vital indicator of person's health [12-14]. A vide variety of sensor devices are available that record oral, skin, tympanic, and rectal mucosa temperatures. These clinical thermometers are based on the variety of transducers (e.g., thermistors, thermocouples, thermopile, semiconductor temperature sensors, P-N junction diode, Resistance Temperature Detectors (RTDs), optical fibre devices, infrared radiation sensors, and liquid crystal temperature sensors).

\section{Magnetic Sensors}

Magnetic sensors are mainly based on the magnetic moment of magnetic material that change the magnetic field or 
temperature, or cause mechanical stress [15]. These variations are measured using sensors. In biomedical applications, magnetic sensors are utilized to hyperthermia treatments or for drug delivery. These applications may include blood, cerebrospinal fluid, culture medium, and organic tissues. Some of the biomedical applications are listed bellow.

(i) Cell culture measurement in in situ

(ii) Test of blood coagulation and blood flow

(iii) Early detection of heart valve bio prostheses failure

(iv) Cancer cell (hyperthermia HeLa cell) treatment with silica coated manganese oxide $\left(\mathrm{MnO}_{2}\right)$ nanoparticles

(v) Endoluminal artificial urinary sphincter

III.

\section{CONCLUSION}

This paper discussed the different physical sensors used in biomedical applications. Several types of physical sensors are used in biomedical applications, such as blood pressure, muscle displacement, blood flow, core/external body temperature, bone growth, and cerebrospinal fluid pressure measurements. Among most of the physical sensors, optical sensors are mostly used in the biomedical applications. Physical sensors are frequently used in the electronic instruments, such as X-ray tomography, PET, ultrasonography, MRI, and measurement of blood flow/pressure, and body temperature. However, researchers are working to develop more sensitive, reliable, stabile and low-cost sensors. Miniaturization of the sensors is a hot topic, which is currently done using semiconductor fabrication methods of microelectronics. The main advantage of fabricating microelectronics is their small size that allows the combining of several sensors on a single chip. In addition, there is progress towards fabrication of better physical sensors with advanced sensing materials and conventional electronics devices.

\section{ACKNOWLEDGMENT}

The research reported in this publication was supported by funding from King Abdullah University of Science and Technology.

\section{REFERENCES}

1. Gungun Lin, Denys Makarov and Oliver G. Schmidtad, "Magnetic Sensing Platform Technologies for Biomedical Applications" Lab Chip, Volume 17, pp. 1884-1912, 2017.

2. Hatice Ceylan Koydemir and Aydogan Ozcan, "Wearable and Implantable Sensors for Biomedical Applications" Annual Review of Analytical Chemistry, Volume 11, pp. 6.1-6.20, 2018.

3. Kenry, Joo Chuan Yeo and Chwee Teck Lim, "Emerging Flexible and Wearable Physical Sensing Platforms for Healthcare and Biomedical Applications", Microsystems \& Nanoengineering, Volume 2, Article number: 16043, 2016.

4. Gabor Harsanyi, Sensors in Biomedical Applications: Fundamentals, Technology and Applications, 1st ed., CRC Press, 2000, 368 Pages.

5. Anindya Nag, Nasrin Afasrimanesh, Shilun Feng and Subhas Chandra Mukhopadhyay, "Strain Induced Graphite/PDMS Sensors for Biomedical Applications" Sensors and Actuators A: Physical, Volume 271, pp. 257-269, 2018.

6. Atika Arshad, Kushsairy Abdul Kadir, Sheroz Khan, A.H.M Zahirul Alam and Rumana Tasnim, "A Pressure Sensor Based Inductive Transducer Designed for Biomedical Applications" 2015 IEEE,
Putrajaya, Malaysia [3rd International Conference on Smart Instrumentation, Measurement and Applications (ICSIMA)].

7. R. Correia, S. James, S.-W. Lee, S. P. Morgan and S. Korposh, "Biomedical Application of Optical Fibre Sensors" Journal of Optics, Volume 20, Number 7, 073003 (25pp), 2018.

8. Yiyan Peng, Yuri M. Shkel and Timothy J. Hall, "A Tactile Sensor for Ultrasound Imaging Systems" IEEE Sensor Journal, Volume 16, pp. 1044-1053, 2016.

9. Hongliang Zhou, Weibin Lin, Xiaocheng Ge and Jian Zhou, "A NonIntrusive Pressure Sensor by Detecting Multiple Longitudinal Waves" Sensors (Basel), Volume 16, pp. 1237, 2016.

10. Ezzat G. Bakhoum and Marvin H. M. Cheng, "Novel Capacitive Pressure Sensor" Journal of Microelectromechanical Systems, Volume 19, pp. 443-450, 2010.

11. Chia-Chu Chiang, Chou-Ching $\mathrm{K}$ Lin and Ming-Shuang Ju, An Implantable Capacitive Pressure Sensor for Biomedical Applications" Sensors and Actuators A-Physical, Volume 134, pp. 382-388, 2007.

12. Carina Barbosa Pereira, Michael Czaplik, Vladimir Blazek, Steffen Leonhardt and Daniel Teichmann, "Monitoring of Cardiorespiratory Signals Using Thermal Imaging: A Pilot Study on Healthy Human Subjects" Sensors, Volume 18, Article number 1541, 2018.

13. Fahimeh Dehkhoda, Ahmed Soltan, Nikhil Ponon, Andrew Jackson, Anthony O'Neill and Patrick Degenaar, "Self-sensing of Temperature Rises on Light Emitting Diode Based Optrodes" Journal of Neural Engineering, Volume 15, Article number 026012, 2018.

14. E. Hemmer, P. Acosta-Mora, J. Méndez-Ramos and S. Fischer, Optical Nanoprobes for Biomedical Applications: Shining a Light on Upconverting and Near-infrared Emitting Nanoparticles for Imaging, Thermal Sensing, and Photodynamic Therapy", Journal of Materials Chemistry B, Volume 5, pp. 4365-4392, 2017.

15. Dieter Suess, Anton Bachleitner-Hofmann, Armin Satz, Herbert Weitensfelder, Christoph Vogler, Florian Bruckner, Claas Abert, Klemens Prügl, Jürgen Zimmer, Christian Huber, Sebastian Luber, Wolfgang Raberg, Thomas Schrefl and Hubert Brückl, "Topologically Protected Vortex Structures for Low-noise Magnetic Sensors with High Linear Range", Nature Electronics, Volume 1, pp. 362-370, 2018.

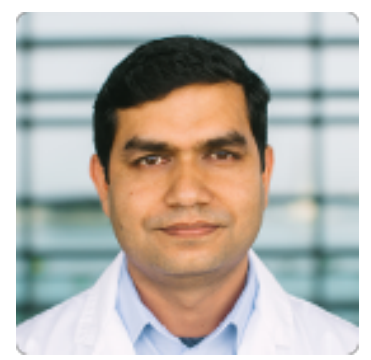

Rafiq Ahmad is a Postdoctoral Fellow of Electrical engineering department at King Abdullah University of Science and Technology (KAUST). He received his Ph.D. from Chonbuk National University in Department of BIN Fusion Technology in 2014. He was postdoctoral researcher in School of Chemical Engineering (March 2014 to Feb 2017) at Chonbuk National University. Then, he worked as contract professor in School of Chemical Engineering (March 2017 to Feb 2018), Chonbuk National University, South Korea. He is currently working in the forefront area of Materials Science with applications focused on design of novel chemical and biological sensors. He is the author of nearly 60 manuscripts.

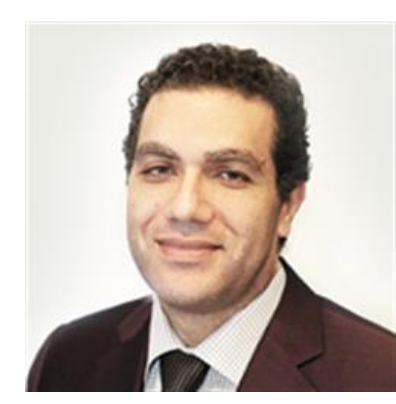

Khaled Nabil Salama is a Professor and founding chair member in the Electrical engineering department at King Abduallah University of Science and Technology. He received his $\mathrm{PhD}$ in electrical engineering from Stanford University in 2005. He is a prominent researcher in the field of memristor-based devices. He is currently working on applications of 
modern electrical analog circuits. He has been active and highly successful in bringing multiple inter-disciplinary fields in biology, microwave engineering, chemistry, material sciences, and mechanical engineering with electrical engineering. He works on application of modern devices to build biomedical sensors. Professor Salama publishes regularly in IEEE Transactions. He is the 4th highest cited author in the area of memristors. He has 15 patents ( 9 at KAUST) in his name and has founded two startups. He had served as Associate Editor on two premier journals, the IEEE Transactions on Circuits and Systems and the IEEE Transactions on Biomedical Circuits and Systems. He also served as a technical program committee member on leading conferences such as IEEE VLSI, IEEE Sensors and IEEE ISCAS. Professor Salama is a senior member of the IEEE. 